\title{
Characteristics of medium- and large-scale TIDs over Japan derived from OI 630-nm nightglow observation
}

\author{
Minoru Kubota ${ }^{1}$, Hiroshi Fukunishi ${ }^{2}$, and Shoichi Okano ${ }^{3}$ \\ ${ }^{1}$ Communications Research Laboratory, Koganei-shi, Tokyo 184-8795, Japan \\ ${ }^{2}$ Department of Geophysics, Graduate School of Science, Tohoku University, Sendai 980-8578, Japan \\ ${ }^{3}$ Planetary Plasma and Atmospheric Research Center, Graduate School of Science, Tohoku University, Sendai 980-8578, Japan
}

(Received July 4, 2000; Revised January 26, 2001; Accepted March 19, 2001)

\begin{abstract}
A new optical instrument for studying upper atmospheric dynamics, called the Multicolor All-sky Imaging System (MAIS), has been developed. The MAIS can obtain all-sky images of airglow emission at two different wavelengths simultaneously with a time resolution of several minutes. Since December 1991, imaging observations with the MAIS have been conducted at the Zao observatory $\left(38.09^{\circ} \mathrm{N}, 140.56^{\circ} \mathrm{E}\right)$. From these observations, two interesting events with wave structures have been detected in OI 630-nm nightglow images. The first event was observed on the night of June 2/3, 1992 during a geomagnetically quiet period. Simultaneous data of ionospheric parameters showed that they are caused by propagation of the medium-scale traveling ionospheric disturbance (TID). Phase velocity and horizontal wavelength determined from the image data are $45-100 \mathrm{~m} / \mathrm{s}$ and $\sim 280 \mathrm{~km}$, and the propagation direction is south-westward. The second event was observed on the night of February 27/28, 1992 during a geomagnetic storm. It is found that a large enhancement of OI 630-nm emission is caused by a propagation of the large-scale TID. Meridional components of phase velocities and wavelengths determined from ionospheric data are 305-695 m/s (southward) and 930-5250 km. The source of this large-scale TID appears to be auroral processes at high latitudes.
\end{abstract}

\section{Introduction}

The existence of atmospheric gravity waves (AGWs) in the Earth's upper atmosphere was originally inferred by Hines (1960) from ground based observations of traveling ionospheric disturbances (TIDs). Gravity waves and their effects seem to be present in the thermosphere at all times and in all geographic locations. There appear to be many sources which contribute to the gravity wave spectrum in the thermosphere. A review of the theoretical and observational results describing AGW/TID phenomena at high latitudes was presented by Hunsucker (1982). He classified TIDs into two types: large-scale TIDs and medium-scale TIDs. TIDs are often generated by high latitude thermospheric forcing due to auroral processes. The propagation and attenuation of these waves have important consequences; for example, on the energy budget of the lower thermosphere.

The OI 630-nm airglow is an important indicator of thermospheric processes, and variations of OI 630-nm intensity were shown to be associated with ionospheric disturbance (Barbier, 1959; Barbier and Glaume, 1962). Since then, observations of the OI 630-nm airglow have been made by many investigators in order to find a possible connection with the TID. Porter et al. (1974) made a theoretical analysis of TIDs and the OI 630-nm emission in mid-latitudes, and they showed that if reasonable values of the ionospheric parameters (as well as a gravity wave with a period of approximately 3 hours) are assumed, the expected fluctuations in the OI

Copy right (C) The Society of Geomagnetism and Earth, Planetary and Space Sciences (SGEPSS); The Seismological Society of Japan; The Volcanological Society of Japan; The Geodetic Society of Japan; The Japanese Society for Planetary Sciences. 630-nm airglow are consistent with observation data. Sobral et al. (1978) measured the OI 630-nm airglow at Arecibo $\left(29^{\circ} \mathrm{N}\right.$ geomagnetic latitude) by using a scanning photometer. They observed descents of the OI 630-nm emission layer, and these descents were associated with the descents of the equatorial ionosphere observed from ionogram data. They also observed occasional enhancements of the emission intensity due to traveling ionospheric disturbances.

On the other hand, TIDs at mid-latitudes have been studied by using HF-Doppler measurement (Shibata, 1986; Waldock and Jones, 1987; Kainuma et al., 1992), and ionospheric parameters $\left(h^{\prime} F\right.$ and $\left.f_{0} F_{2}\right)$ measurement (Hajkowicz, 1990, 1991). It is evident that surges in the auroral electrojet generate trains of large-scale TIDs in both hemispheres, and these trains subsequently propagate equatorward causing substantial height enhancements of the ionosphere.

Using CCD detectors to measure the nightglow enables us to observe two-dimensional nightglow structures. Mendillo et al. (1997) conducted 630-nm imaging observations at Arecibo, Puerto Rico, by using an all-sky, image-intensified CCD camera system. They observed several structures in the OI 630-nm airglow: gravity wave signatures; a brightness wave passage in the OI $630-\mathrm{nm}$ airglow (which is linked to meridional winds driven by the midnight temperature maximum pressure bulge); and OI 630-nm airglow depletion patterns associated with equatorial spread $F$ instabilities. In 1996, using a monochromatic imager, Taylor et al. (1998) observed "wave-like" structures in the OI 630-nm thermospheric nightglow emission over Japan. A strong tendency for wave progression towards the southwest was found on all 
but one occasion (total of five events). The 630-nm waveforms were dissimilar to spread $F$ related depletions in terms of both their visual characteristics and their expected fieldaligned orientation.

A new optical instrument, called the Multicolor All-sky Imaging System (MAIS), has been developed at Tohoku University to study the atmospheric dynamics in the region from the mesopause to the thermosphere. With the MAIS, we simultaneously observed a 630-nm emission and a 608-nm background emission at the Zao observatory of the Upper Atmosphere and Space Research Laboratory, Tohoku University in Japan, and we derived the absolute intensity distributions of the OI 630-nm nightglows. Two major events appeared in the OI 630-nm nightglow: a "wave-like" event, which has a close resemblance to the phenomena observed by Taylor et al. (1998), and a large enhancement of the OI 630nm intensity caused by a propagation of the large-scale TID. In this work, we have investigated the relation of these events with ionospheric disturbances observed by the ionosonde network over Japan.

\section{Instrumentation}

A schematic diagram of the MAIS is shown in Fig. 1. Allsky images of airglow emissions at two different wavelengths can be simultaneously obtained by using a prism and semicircular interference filters. A detailed description to explain how these all-sky images with different wavelengths are adjacently produced is given as follows. As shown in Fig. 2, rays $\mathbf{p}$ and $\mathbf{q}$ emitted from all-sky image $\mathbf{I}$ are collimated by the collimator lens. The direction of the collimated beam, which passed through the semicircular interference filter $\mathbf{A}$, is slightly bent inward by the right side of the prism in the figure. The collimated beam is then focused by the imaging lens to point $\mathbf{a}_{A}$ on the focal plane. Similarly, the rays emitted from $\mathbf{b}$ and $\mathbf{c}$ on the all-sky image $\mathbf{I}$ and passed through the filter $\mathbf{A}$ are focused to points $\mathbf{b}_{A}$ and $\mathbf{c}_{A}$, respectively. In this way, a monochromatic all-sky image with the wavelength of filter $\mathbf{A}$ is produced on the left side of the optical axis. Likewise, the rays emitted from $\mathbf{a}, \mathbf{b}$, and $\mathbf{c}$ on the all-sky image $\mathbf{I}$ and passed through filter $\mathbf{B}$ are focused to points $\mathbf{a}_{B}, \mathbf{b}_{B}$, and $\mathbf{c}_{B}$, respectively, to produce an all-sky image with the wavelength of filter $\mathbf{B}$ on the right side of the optical axis. In this manner, two monochromatic all-sky images with different wavelengths are produced adjacently. The diameter of each monochromatic all-sky image is $6 \mathrm{~mm}$.

The image intensifier (II) on which the monochromatic allsky images are focused is a highly sensitive, two-dimensional photon detector (Model V2697U, Hamamatsu Photonics Corp.). This II has a single-stage microchannel plate (MCP) and its nominal spatial resolution is $35 \mu \mathrm{m}$. This limitation in image resolution should correspond to an angular resolution of about $1^{\circ}$ because the diameter of an all-sky $\left(180^{\circ}\right.$ FOV) image is $6 \mathrm{~mm}$. An image on the phosphor output screen of the II is refocused on the surface of a cooled CCD chip which has $1000 \times 1018$ pixels with the size of each pixel being $12 \mu \mathrm{m} \times 12 \mu \mathrm{m}$. The ratio of reduction in image size is $1: 1$. The cooled CCD camera is a model C3640 manufactured by Hamamatsu Photonics. This detector enables us to obtain airglow images with high time resolution; that is 180 -sec exposure time for OI 630-nm emissions.

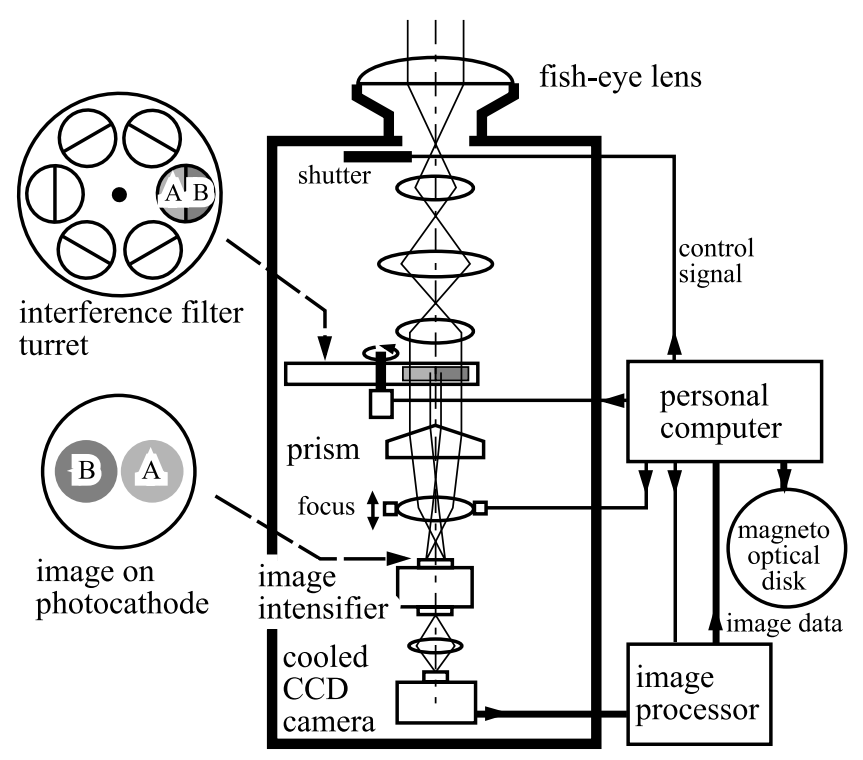

Fig. 1. A system diagram of the MAIS.

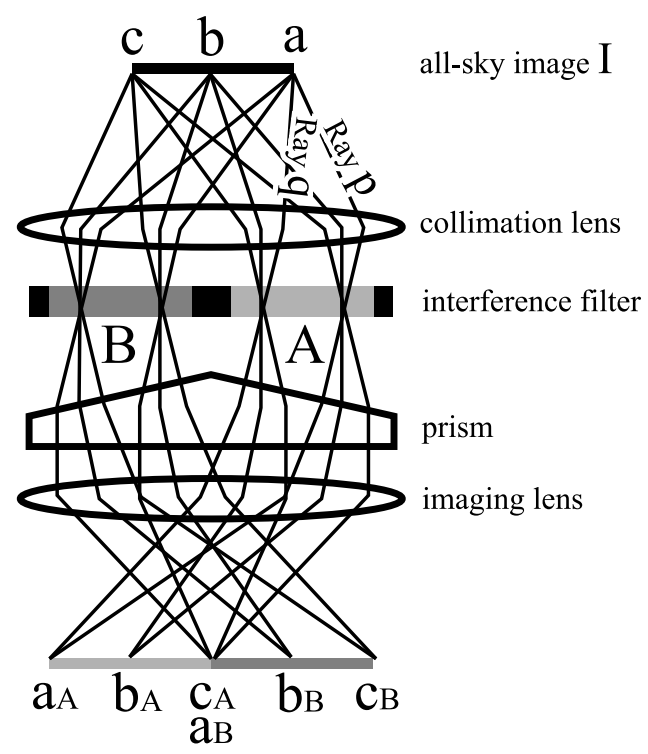

Fig. 2. Optical ray paths that form two adjacent all-sky images at different wavelengths.

The MAIS has six channels, each having a pair of filters with different wavelengths. Therefore, by switching the channels, 12 different wavelengths, including OI 558-nm, OI 630-nm, Na D, and $\mathrm{OH}(8-3)$ emissions, can be measured.

\section{Image Data Processing}

To obtain absolute intensity distributions of airglows, the raw image data observed by MAIS are processed in a sequence as described below.

\subsection{Correction of non-uniform sensitivity over the im- age}

The sensitivity of the MAIS is not uniform over an image due to a non-uniformity in sensitivity of the photocathode of the II and due to the characteristics of the optical system whose transmission is dependent on incident angle. To calibrate the non-uniform sensitivity, a light source, which fills 
the field of view of the MAIS with uniform radiance, was manufactured. The light source is made of a hemispherical acrylic dome with a diameter of $50 \mathrm{~cm}$ and pieces of fluorescent paper stuck on the inner surface of the dome. The inside of the dome is filled with fluorescence radiations for several minutes after irradiation by an incandescent lamp $(100 \mathrm{~W})$ placed at its center. Uniformity of the fluorescence was examined by using a photodiode, and the amount of scatter remained within $5 \%$ over the whole dome. The dome is placed on the fish-eye lens of the MAIS as a light source, and calibration images are taken. An example of the image obtained in this calibration is shown in Fig. 3. The maximum non-uniform sensitivity was about $50 \%$ over the whole image.

\subsection{Correction of background continuum}

The intensity of background emission $I_{\mathrm{bg}}(\lambda)$ [Rayleigh/ $n m$ ] is assumed to be the same at both the wavelengths of an airglow channel (around $630 \mathrm{~nm}$ ) and of the corresponding background channel (around $608 \mathrm{~nm}$ ); that is,

$$
I_{\mathrm{bg}}(\lambda)=I_{\mathrm{bg}}^{\circ} .
$$

The width of an airglow emission line is much narrower than the equivalent width of a filter. The airglow emission $I_{\mathrm{ag}}(\lambda)[$ Rayleigh] can therefore be approximated by a $\delta$ function;

$$
I_{\mathrm{ag}}(\lambda)=I_{\mathrm{ag}}^{\circ} \cdot \delta\left(\lambda-\lambda_{\mathrm{ag}}\right) .
$$

When airglow is imaged at channel $\alpha$ and background is imaged at channel $\beta$, their number of counts, $N_{\alpha}$ and $N_{\beta}$, are given by

$$
\begin{aligned}
N_{\alpha}= & k_{\alpha}^{\circ} \cdot E \cdot T_{\alpha} \int_{0}^{\infty}\left(I_{\mathrm{ag}}^{\circ} \cdot \delta\left(\lambda-\lambda_{\mathrm{ag}}\right)+I_{\mathrm{bg}}^{\circ}\right) \\
& \cdot F_{\alpha}(\lambda) \cdot S(\lambda) \cdot d \lambda \\
= & k_{\alpha}^{\circ} \cdot E \cdot T_{\alpha} \cdot\left\{I_{\mathrm{ag}}^{\circ} \cdot F_{\alpha}\left(\lambda_{\mathrm{ag}}\right) \cdot S\left(\lambda_{\mathrm{ag}}\right)\right. \\
& \left.+I_{\mathrm{bg}}^{\circ} \cdot \int_{0}^{\infty} F_{\alpha}(\lambda) \cdot S(\lambda) \cdot d \lambda\right\}
\end{aligned}
$$

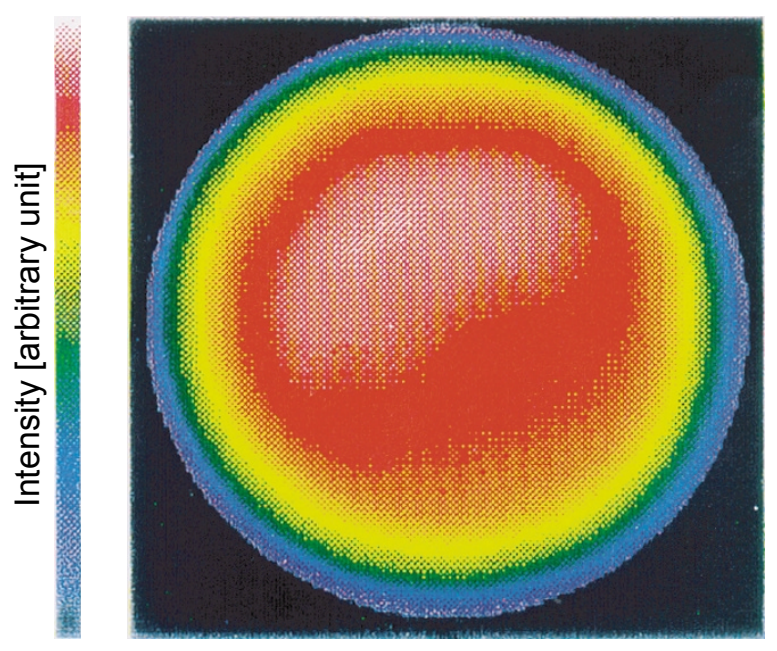

Fig. 3. An example of image obtained with the fluorescent dome as a light source. This image is used for the flat field sensitivity calibration.

$$
\begin{aligned}
N_{\beta} & =k_{\beta}^{\circ} \cdot E \cdot T_{\beta} \int_{0}^{\infty} I_{\mathrm{bg}}^{\circ} \cdot F_{\beta}(\lambda) \cdot S(\lambda) \cdot d \lambda \\
& =k_{\beta}^{\circ} \cdot E \cdot I_{\mathrm{bg}}^{\circ} \cdot T_{\beta} \int_{0}^{\infty} F_{\beta}(\lambda) \cdot S(\lambda) \cdot d \lambda,
\end{aligned}
$$

where $k_{\mathrm{ch}}^{\circ}$ [counts /sec.Rayleigh] is the sensitivity to be determined for each channel using a standard brightness plate whose spectral radiances exactly known. E[sec $]$ is the exposure time, $T_{\mathrm{ch}}$ is the total transmission function (which depends on the optical components of the MAIS), $F_{\text {ch. }}(\lambda)$ is the filter transmission function, and $S(\lambda)$ is the quantum efficiency of the image intensifier. The airglow intensity $I_{\mathrm{ag}}^{\circ}[$ Rayleigh $]$ is therefore given by

$$
\begin{aligned}
I_{\mathrm{ag}}^{\circ}= & \frac{N_{\alpha}}{k_{\alpha}^{\circ} \cdot E \cdot T_{\alpha} \cdot F_{\alpha}\left(\lambda_{\mathrm{ag}}\right) \cdot S\left(\lambda_{\mathrm{ag}}\right)} \\
& -\frac{N_{\beta} \int_{0}^{\infty} F_{\alpha}(\lambda) \cdot S(\lambda) \cdot d \lambda}{k_{\beta}^{\circ} \cdot E \cdot T_{\beta} \cdot F_{\alpha}\left(\lambda_{\mathrm{ag}}\right) \cdot S\left(\lambda_{\mathrm{ag}}\right) \int_{0}^{\infty} F_{\beta}(\lambda) \cdot S(\lambda) \cdot d \lambda} .
\end{aligned}
$$

Using Eq. (5), we can correct the background continuum, and obtain the absolute intensity of airglow.

\subsection{Correction of the van Rhijn effect and atmospheric extinction}

If the thickness of the airglow emission layer is negligible compared to the earth radius $R_{E}$, the airglow intensity is accepted to be proportional to the length of the line of sight in the emission layer. The intensity at zenith angle $\theta, I(\theta)$ is given by

$$
\begin{aligned}
I(\theta) & =\left[1-\left(\frac{R_{E}}{R_{E}+h_{a g}}\right)^{2} \sin ^{2} \theta\right]^{-1 / 2} \cdot I(0) \\
& =V\left(h_{a g}, \theta\right) \cdot I(0),
\end{aligned}
$$

where $I(0)$ is the intensity at zenith, $h_{a g}$ is the altitude of the airglow emission layer, and $V(h, \theta)$ is called the van Rhijn correction factor (Tohmatsu and Ogawa, 1990). Airglow intensities observed from the ground are also affected by atmospheric extinction. For cloud-free conditions, the atmospheric extinction at visible wavelengths is mainly caused by the following factors: Rayleigh scattering by atmosphere, continuum absorption and scattering by aerosols, and continuum absorption by $\mathrm{O}_{3}$ (Arthur, 2000). The atmospheric extinction is a very complex phenomenon, however we correct it using the following simple techniques. The relation of the apparent airglow intensity $I(\theta)$ at the ground to the true airglow intensity $I_{\text {true }}(\theta)$ at the emission layer is given by

$$
I(\theta)=I_{\text {true }}(\theta) \cdot 10^{-0.4 a F(\theta)}
$$

and

$$
\begin{aligned}
F(\theta)= & {[\cos \theta+0.15} \\
& \left.\cdot\left(93.885-\theta \cdot \frac{180}{\pi}\right)^{-1.253}\right]^{-1},
\end{aligned}
$$

where $F(\theta)$ is an empirical equation that shows air mass at zenith angle $\theta$ (Kasten, 1966), and $a$ is the atmospheric extinction coefficient. The van Rhijn correction factor combined with atmospheric extinction at certain $a$ values is plotted in Fig. 4. The van Rhijn correction factor is calculated by 


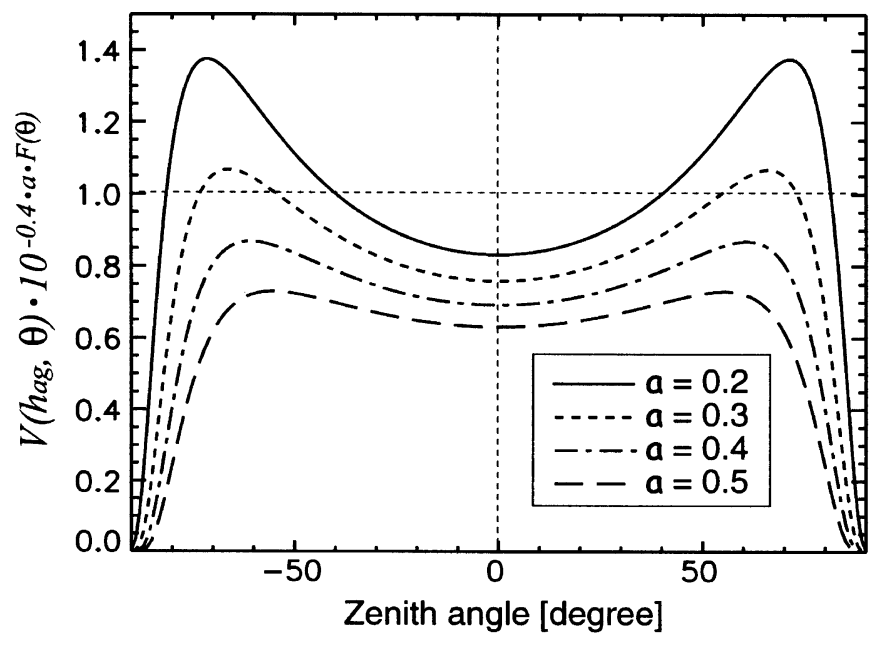

Fig. 4. Variation of the van Rhijn correction factor combined with atmospheric extinction versus zenith angle.

assuming that the emission heights of OI 630-nm emission is $250 \mathrm{~km}$. Because $a$ varies with atmospheric conditions at the time of observation, we need to determine $a$ for every observation. Assuming that $a$ is constant over a one night and that an average of all images obtained in one night gives an uniform intensity distribution, we determine $a$ as follows: (1) images obtained in one night are averaged in order to obtain a single image; (2) a curve representing the intensity change against zenith angle is derived from the averaged image; and (3) the curve obtained in the previous step is compared with the profiles in Fig. 4. Then the value of $a$ is determined from the profile that gives the best fit. In most cases, the value of $a$ determined in this way is about 0.4 .

\subsection{Coordinates transformation}

The fish-eye lens images are transformed to an image in geographic coordinates by assuming an altitude of the airglow emission layer. The relationship between the fish-eye coordinates (expressed by the zenith angle $\theta$ and the azimuth) and the geographic coordinates (expressed by the horizontal distance $r$ from the observatory and the azimuth) is given geometrically by

$$
r=R_{E} \cdot \alpha
$$

and

$$
\alpha=\theta-\sin ^{-1}\left(\frac{R_{E} \cdot \sin \theta}{R_{E}+h_{\mathrm{ag}}}\right),
$$

where $R_{E}$ is the earth radius and $h_{\mathrm{ag}}$ is the altitude of airglow emission layer. By this coordinates transformation, it becomes easier to measure direction and velocity of movements of airglow structures. Comparisons between airglow intensity distribution and the ionospheric data obtained at other locations also become possible.

Figure 5 shows an example of the image format of the OI 630-nm emission after being subjected to the processing described above. The height of airglow emission layer is assumed to be $250 \mathrm{~km}$. A square area, whose center corresponds to the zenith of the Zao observatory and whose side span is approximately $1150 \mathrm{~km}$, is cut out of an image. The time denotes the start time of exposure, and this particular image was obtained with a 180-second exposure between 2125 JST (Japanese Standard Time; JST $=\mathrm{UT}+9 \mathrm{hr}$.) and

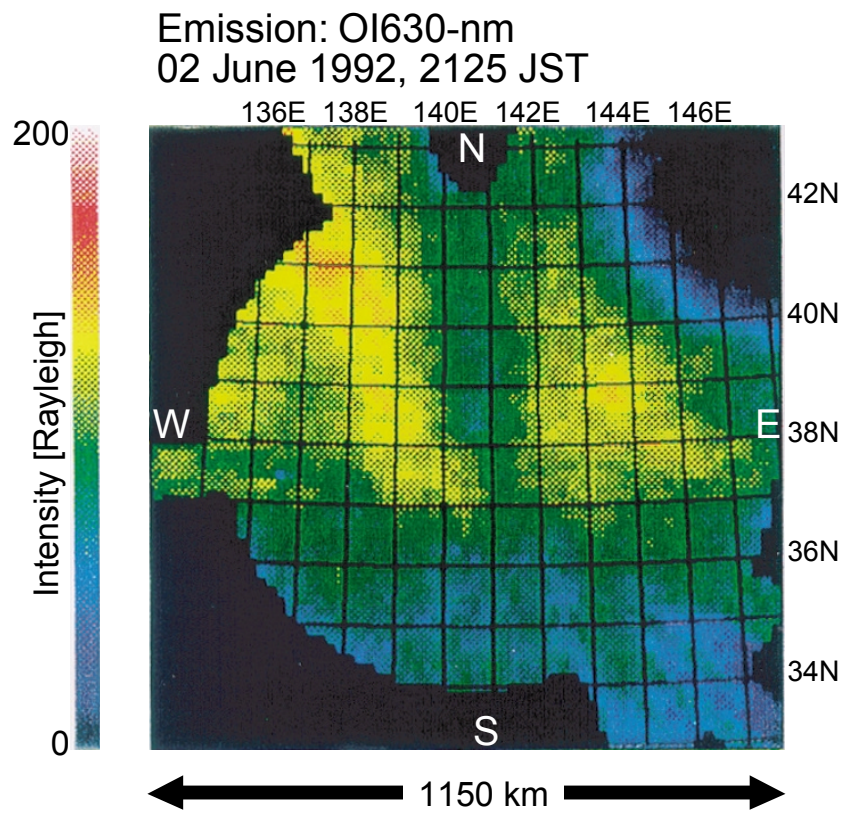

Fig. 5. An example of an OI 630-nm airglow image. The span of one side is approximately $1150 \mathrm{~km}$. The upside and the right side of the image correspond to the geographic north and east, respectively. Shadowed area in this image is due to obstructive nearby trees and surrounding mountains. Vertical and horizontal lines in the image are longitudinal and latitudinal lines at 1-degree intervals, and the center of the image corresponds to the zenith of the Zao observatory $\left(38^{\circ} 5.6^{\prime} \mathrm{N}, 140^{\circ} 33.7^{\prime} \mathrm{E}\right)$.

2128 JST on June 2, 1992. Shadowed areas in this image are due to obstacles, nearby trees and surrounding mountains. The edge on the northeast side is contaminated by scattered city light of Sendai. These factors must be taken these into consideration when we discuss on the intensity distribution of the OI 630-nm emission.

\section{Observational Results}

The instrument was installed at the Zao observatory in December 1991, and observations of nightglows have been carried out on clear nights during new moon periods. The geographic location of the site is $38.09^{\circ} \mathrm{N}$ and $140.56^{\circ} \mathrm{E}$ at 


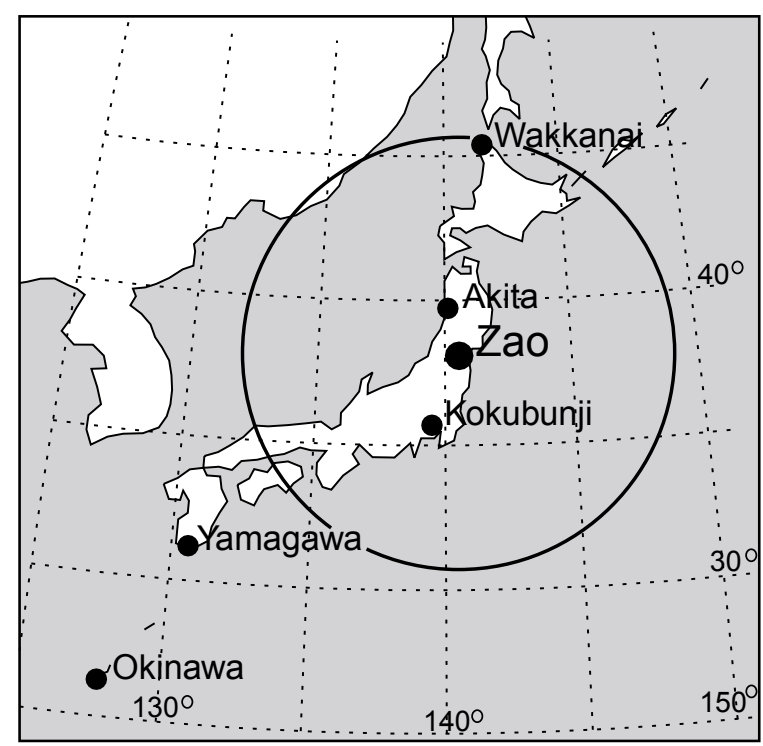

Fig. 6. A map of Japan with an all-sky imaging coverage. The circle with a radius of $800 \mathrm{~km}$ is the coverage of the OI $630-\mathrm{nm}$ emission at an assumed height of $250 \mathrm{~km}$. Wakkanai, Akita, Kokubunji, Yamagawa, and Okinawa are ionospheric observatories. an altitude of $410 \mathrm{~m}$. Figure 6 shows the imaging coverage of the actual MAIS and locations of the other ionospheric observatories (Wakkanai, Akita, Kokubunji, Yamagawa, and Okinawa).

The observations were made on 13 nights in 1992. However, only the results from two specific nights when TID events were observed in the OI 630-nm emission are given here. These events occurred on the night of June 2/3, 1992 during a geomagnetically quiet period and on the night of February 27/28, 1992 during a geomagnetic storm.

\subsection{Wave-like structures of OI 630-nm emission during a geomagnetically quiet period}

On the night of June 2/3, 1992, a small substorm occurred at high latitude around $1600 \mathrm{JST}$ but, in general, geomagnetic activity was low and $K p$ index ranged from $1_{-}$to $1_{+}$during the observation period. On this night, OI $630-\mathrm{nm}$ emission images were obtained every 30 minutes from 2030 JST to 0225 JST. And a part of the data are displayed in Fig. 7.

At 2125 JST, a patch-shaped, enhanced emission area appeared around $39^{\circ} \mathrm{N}$ and $143.5^{\circ} \mathrm{E}$ (see Figs. 5 and 7). This area moved south-westward as increasing intensity and reached a location around $37.5^{\circ} \mathrm{N}$ and $141.5^{\circ} \mathrm{E}$ at $2155 \mathrm{JST}$ and further around $36.75^{\circ} \mathrm{N}$ and $139.75^{\circ} \mathrm{E}$ at $2225 \mathrm{JST}$. To
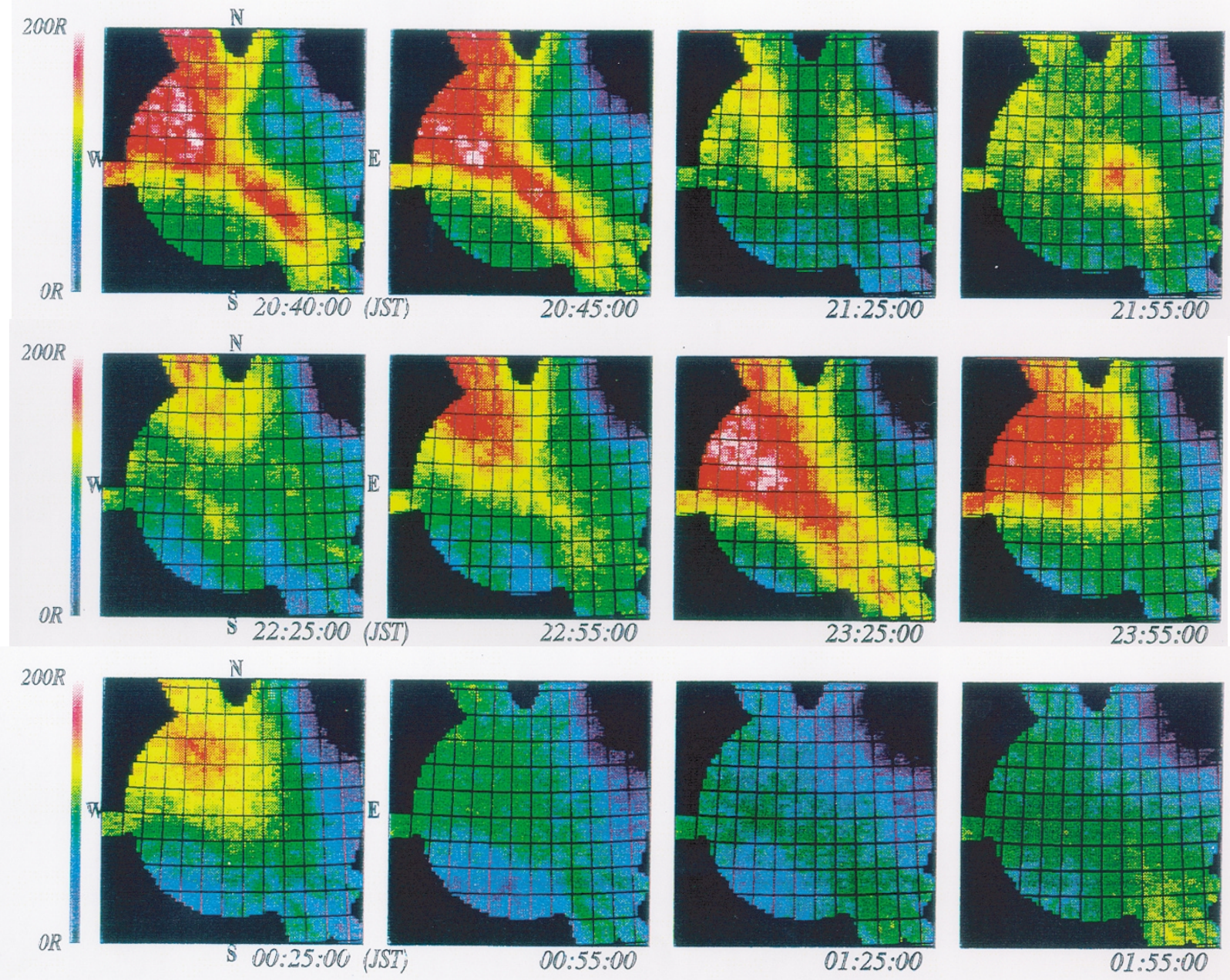

Fig. 7. Images of OI 630-nm airglow on the night of June 2/3, 1992. 
June 2, 1992

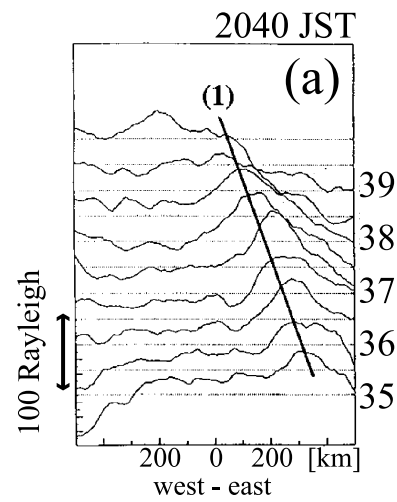

2225 JST

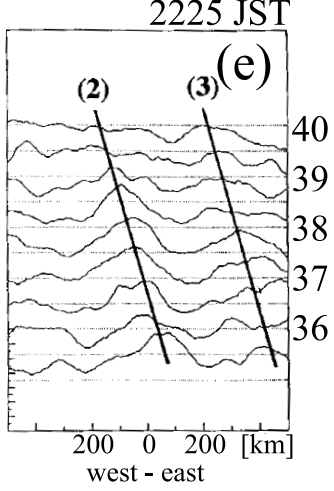

2045 JST

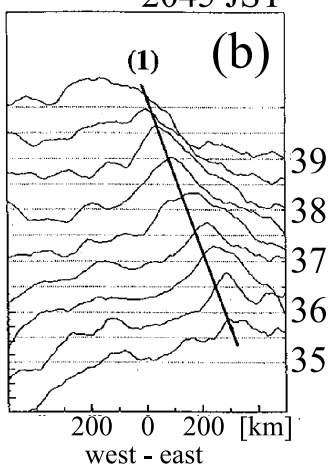

2255 JST

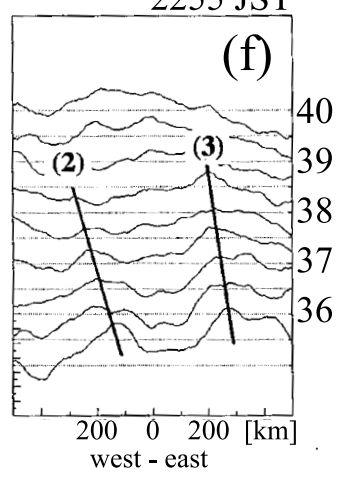

2125 JST

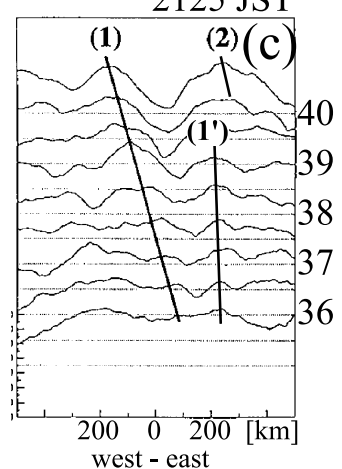

2325 JST

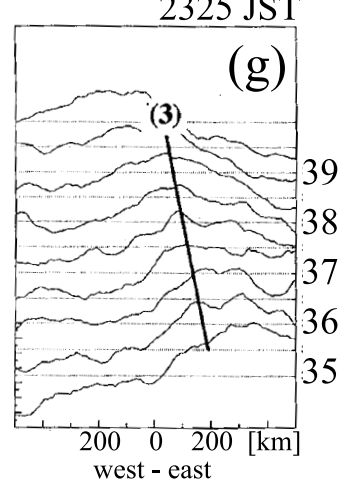

2155 JST

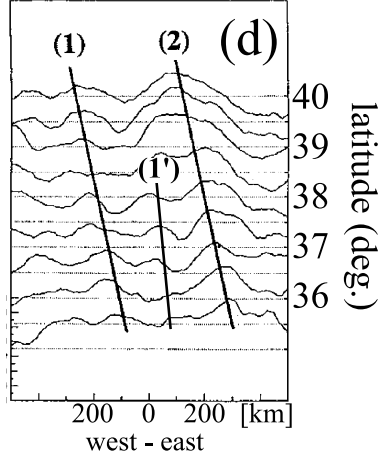

2355 JST

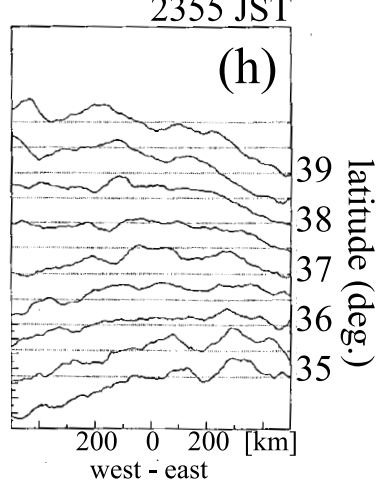

Fig. 8. Longitudinal distributions of OI $630-\mathrm{nm}$ intensity at every $0.5^{\circ}$ intervals in latitude. (a)-(h) correspond to eight consecutive images from 2040 JST to 2355 JST presented in Fig. 7.

see the movement of the enhanced area more clearly, a time series of longitudinal distributions of the OI 630-nm intensity are displayed in Fig. 8 at intervals of $0.5^{\circ}$ for the latitude range of $35^{\circ}-40^{\circ}$. At 2040 JST (Fig. 8(a)), the peaks of longitudinal distributions are found to shift eastward with deceasing latitude as denoted by line (1). The band structure (1) moved westward with time as seen in Figs. 8(b) and (c) and the second big band, denoted as line (2), appeared on the eastside of line (1). It should be noted that the peaks along line (2) correspond to the patch-shaped structure mentioned above. At 2225 JST, the first band structure disappeared and a new one, which is denoted as line (3), appeared on the east side parallel to line (2) as shown in Fig. 8(e). At 2255 JST in Fig. 8(f) these two bands of (2) and (3) moved further westward. At 2325 JST, the band of (2) disappeared as seen in Fig. 8(g), and the band of (3) moved further westward as increasing emission intensity. Finally, no wave structures were identified at 2355 JST (Fig. 8(h)). It should be noted that the wave structures were not uniform. The structures (bands) change their shapes as they propagate. There are also small bands between the big ones, denoted as line (1') in Figs. 8(c) and (d).

The separation in the east-west direction between the two lines of (1) and (2) is about $400 \mathrm{~km}$ at $2155 \mathrm{JST}$ (Fig. 8(d)). The separation between (2) and (3) is also about $400 \mathrm{~km}$ at 2225 JST (Fig. 8(e)). For the coordinates transformation mentioned in Subsection 3.4, we assume that the height of OI 630 -nm emission layer is $250 \mathrm{~km}$. But according to the recent observations, the emission altitude can vary more than $50 \mathrm{~km}$ (Mendillo et al., 1997). It should be noted that an estimation error for the $400 \mathrm{~km}$ separation is about $\pm 30 \mathrm{~km}$, when the actual emission altitude has an ambiguity of $\pm 25 \mathrm{~km}$ from the assumed altitude.

Both Figs. 7 and 8 strongly suggest that waves propagated south-westward. The horizontal wavelength, which corresponds to the band separation in the southwest-northeast direction, is estimated as about $280 \mathrm{~km}$. The phase velocities of the wave fronts (1), (2), and (3) are roughly estimated to be about 70, 100, and $45 \mathrm{~m} / \mathrm{s}$, respectively.

\subsection{Enhancements of OI 630-nm emission during a ge- omagnetic storm}

On the night of February 27/28, 1992, the value of $K p$ index during the period of airglow observation ranged from 5 to $6_{+}$. Imaging observation on this night was made between 2225 and 0300 JST, but it was interrupted from 2315 to 0010 JST because of operation failure of the MAIS. Except for this interruption, concurrent all-sky images of OI 630-nm and OI 558-nm emissions were obtained every 5 minutes.

The all-sky images of OI 630-nm emission from 2305 to 0105 JST are shown in Fig. 9. The emission intensity was relatively low ( $<150$ Rayleigh) until $2310 \mathrm{JST}$, but after that it was remarkably enhanced. At 0010 JST the northern half of the image is more enhanced, and at 0030 JST enhanced area extended southward covering all the field of view. At this time the intensity of the emission reached a maximum (350 Rayleigh), and then it subsided gradually toward the end of the observation (0255 JST).

In the mid-latitude region, the OI 630-nm lines are usually emitted by dissociative recombination of $\mathrm{O}_{2}{ }^{+}$ions. Therefore, variations of the OI 630-nm emission intensity appear 


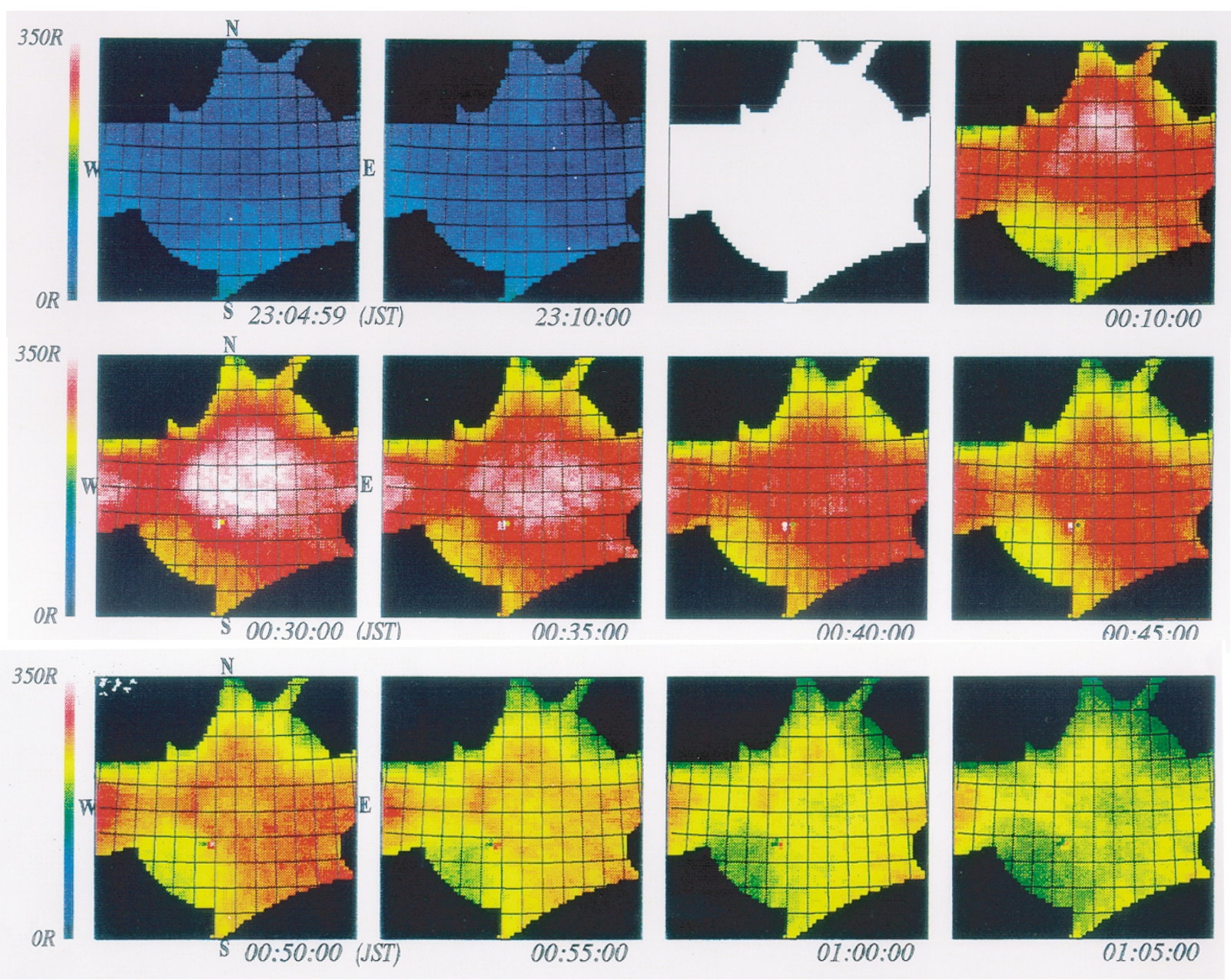

Fig. 9. Images of OI 630-nm airglow on the night of February 27/28, 1992.

to be strongly related to the ionospheric parameters of the $F$ region. The relationship between the intensity variations of OI 630-nm emission and the variations of ionospheric parameters is discussed in more detail in the next section.

\section{Discussions}

\subsection{Wave-like structures of OI 630-nm emission and} medium-scale TID on June 2/3, 1992

On the night of June $2 / 3,1992$, ionospheric parameters, $h^{\prime} F$ and $f_{0} F_{2}$, were obtained every 15 minutes at Wakkanai, Akita, Kokubunji, Yamagawa, and Okinawa ionospheric observatories (operated by Communications Research laboratory (CRL)). These data are plotted in Figs. 10 and 11, respectively. There is no data from Akita after 2315 JST because of operation failure, and there are also occasional missing sections in the data from other observatories. The values of $f_{0} F_{2}$ at all observatories generally decrease during the airglow observation (2040-0230 JST), while those of $h^{\prime} F$ are fluctuating with a period of 1 to 3 hours without obvious coherency among the observatories.

It has been established by many observations that the intensity of the OI 630-nm airglow in the nighttime has a close relation to the electron density and the apparent height of the $F$ region. Barbier (1959) showed that the OI 630-nm intensity is given by an empirical formula using the ionospheric parameters $\left(h^{\prime} F, f_{0} F_{2}\right)$ as

$$
\begin{aligned}
4 \pi I(630.0 \mathrm{~nm})= & A\left(f_{0} F_{2}\right)^{2} \exp \left(-\frac{h^{\prime} F-200}{H}\right) \\
& +B,
\end{aligned}
$$

where $H$ is the scale height of molecular oxygen in $\mathrm{km}, A$ and $B$ are constants determined empirically, and $h^{\prime} F$ and $f_{0} F_{2}$ are respectively the minimum virtual height of the $F$ layer and the ordinary wave critical frequency of the $F_{2}$ layer, related to the electron density of the $F$ region.

Solid lines in Fig. 12 show intensity variations of OI 630$\mathrm{nm}$ emission on June 2/3, 1992 at the zenith of Akita, Zao, and Kokubunji. The OI 630-nm intensities were taken from the image data presented in Fig. 7. Dotted lines show the expected variations of the OI 630-nm intensities calculated from $h^{\prime} F$ and $f_{0} F_{2}$ at Akita and Kokubunji, by using the Barbier's formula. In this calculation, we adopted values of $H$, $A$, and $B$ as $41.5 \mathrm{~km}, 4.14$, and 20.7 , respectively. There is an agreement between the observed and the calculated intensities at the zenith of Kokubunji. Periodic variations in the calculated intensity at Kokubunji are due to the fluctuations in the value of $h^{\prime} F$ as seen in Fig. 10. On the other hand, there are no similar changes in $f_{0} F_{2}$ associated with the electron density of the $F_{2}$ peak. These results indicate that the ionospheric disturbances that cause the wave-like structures 


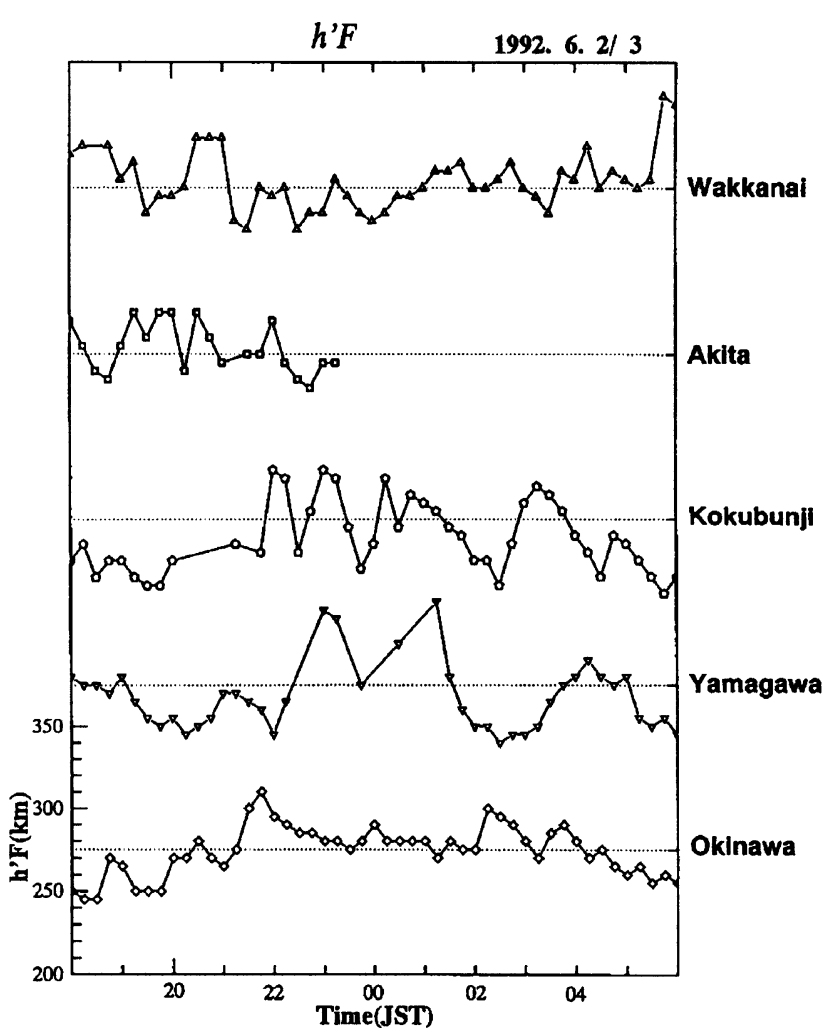

Fig. 10. Variations of $h^{\prime} F$ observed at Wakkanai, Akita, Kokubunji, Yamagawa, and Okinawa on June 2/3, 1992.

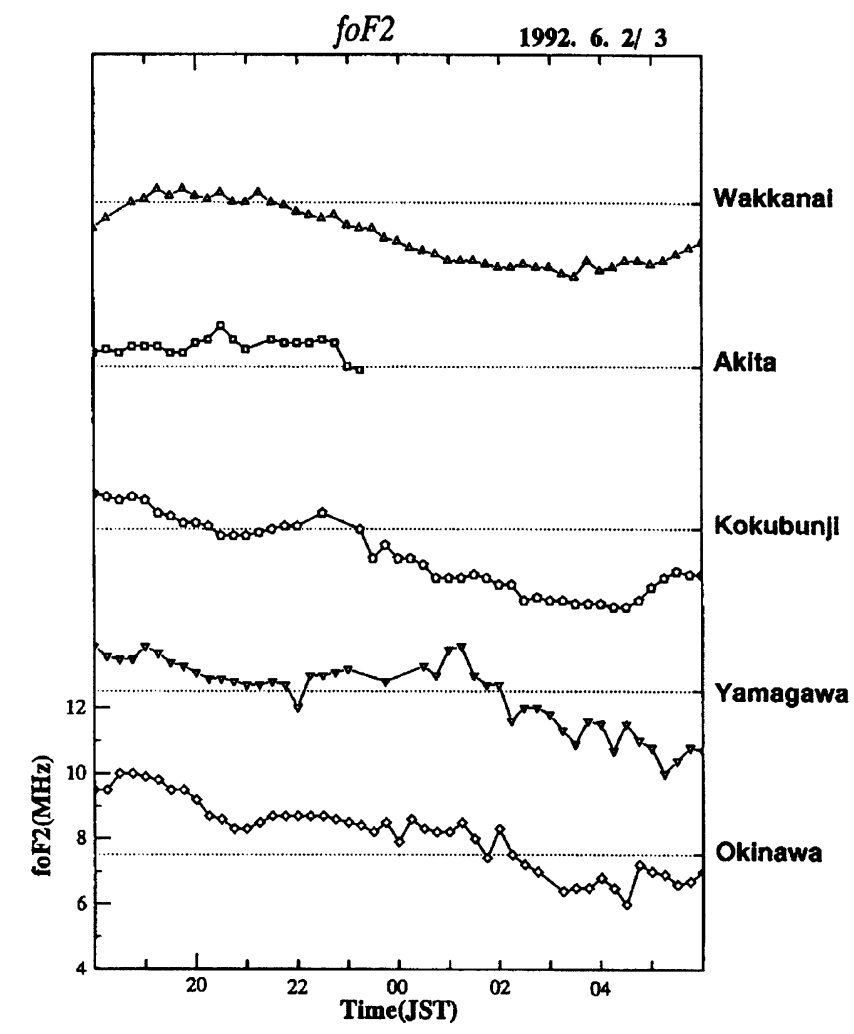

Fig. 11. Variations of $f_{0} F_{2}$ observed at Wakkanai, Akita, Kokubunji, Yamagawa, and Okinawa on June 2/3, 1992.

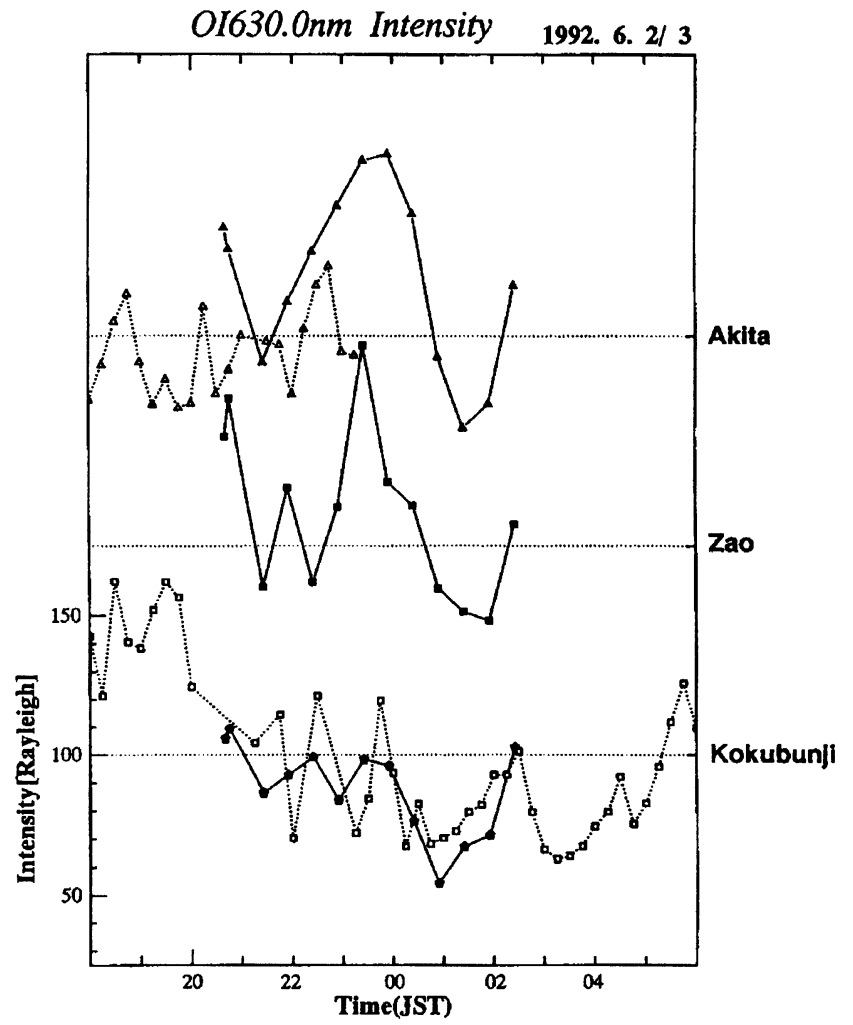

Fig. 12. Variations of OI 630-nm intensity at the zenith of Akita, Zao, and Kokubunji observatories on June 2/3, 1992. Solid lines are the intensities observed with the MAIS. Dotted lines are the values calculated from the ionospheric data using the Barbier's formula.

of the OI 630-nm emission exist in the lower-altitude side of the $F$ region. This ionospheric disturbance can be classified as a medium-scale TID by its phase velocity and wavelength obtained from the imaging observation (Hunsucker, 1982).

\subsection{Remarkable enhancement of OI 630-nm emission} and large-scale TID on February 27/28, 1992

On February 27/28, 1992, OI 630-nm emission showed a remarkable enhancement around midnight as shown in Subsection 4.2. Ionospheric parameters, $h^{\prime} F$ and $f_{0} F_{2}$, taken every 15 minutes at Wakkanai, Akita, Kokubunji, Yamagawa, and Okinawa, are shown in Figs. 13 and 14, respectively. In Fig. 14 , there is a trend that the values of $f_{0} F_{2}$ gradually decrease throughout the night. It is also found that the value of $f_{0} F_{2}$ are coherently fluctuating with a period of 1.5 to 3 hours, and that the phase of the fluctuations shifts from north to south (from Wakkanai to Yamagawa). This phase shift is clearly seen for a large hump in $f_{0} F_{2}$ around midnight at all stations except Okinawa. The values of $h^{\prime} F$ presented in Fig. 13 also fluctuate with a period of 1 to 3 hours, showing a similar southward phase shift. Note that a large decrease in $h^{\prime} F$ occurs around $0100 \mathrm{JST}$. Figure 15 shows the relationship between the intensity variations of OI630-nm emission and the ionospheric parameter changes on February $27 / 28,1992$. Solid lines represent the intensities of OI 630-nm emission between 2225-0300 JST at the zenith of Akita, Zao, and Kokubunji obtained from the MAIS data. On the other hand, dotted lines show calculated intensities using ionospheric parameters measured at Akita and Kokubunji. It 


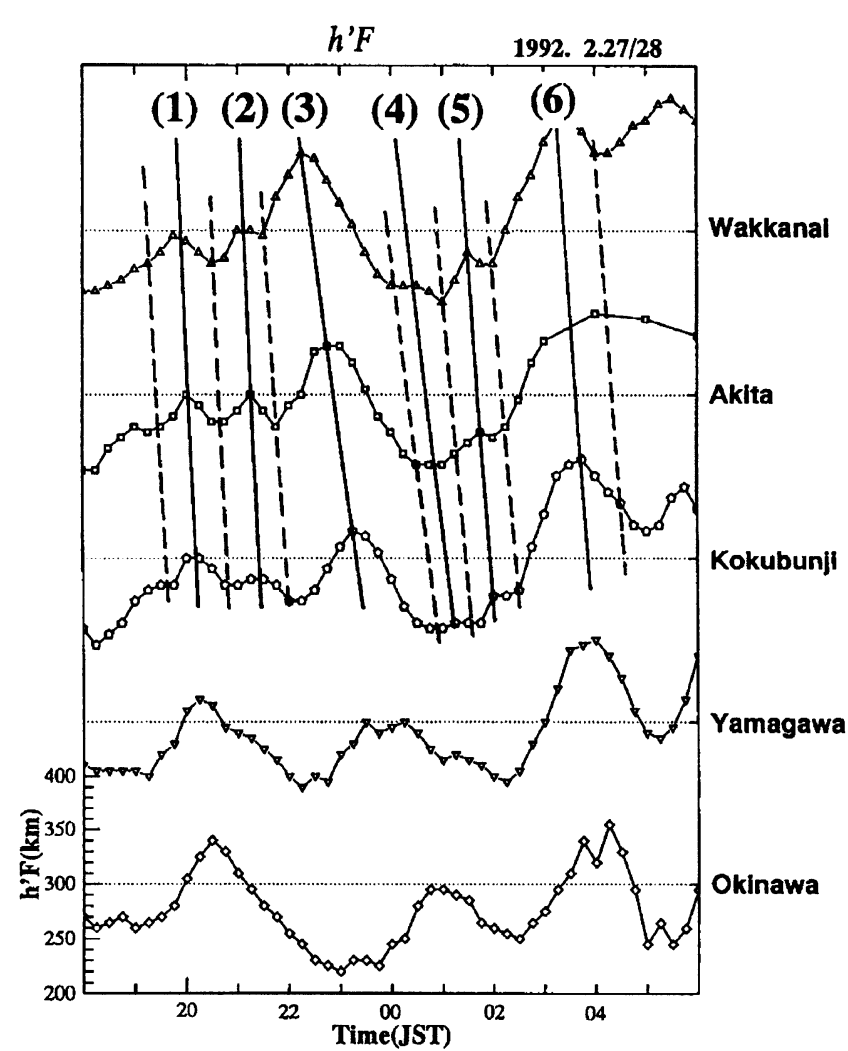

Fig. 13. Variations of $h^{\prime} F$ observed at Wakkanai, Akita, Kokubunji, Yamagawa, and Okinawa on February 27/28, 1992. Solid and dashed lines show phase relations of peaks and valleys, respectively.

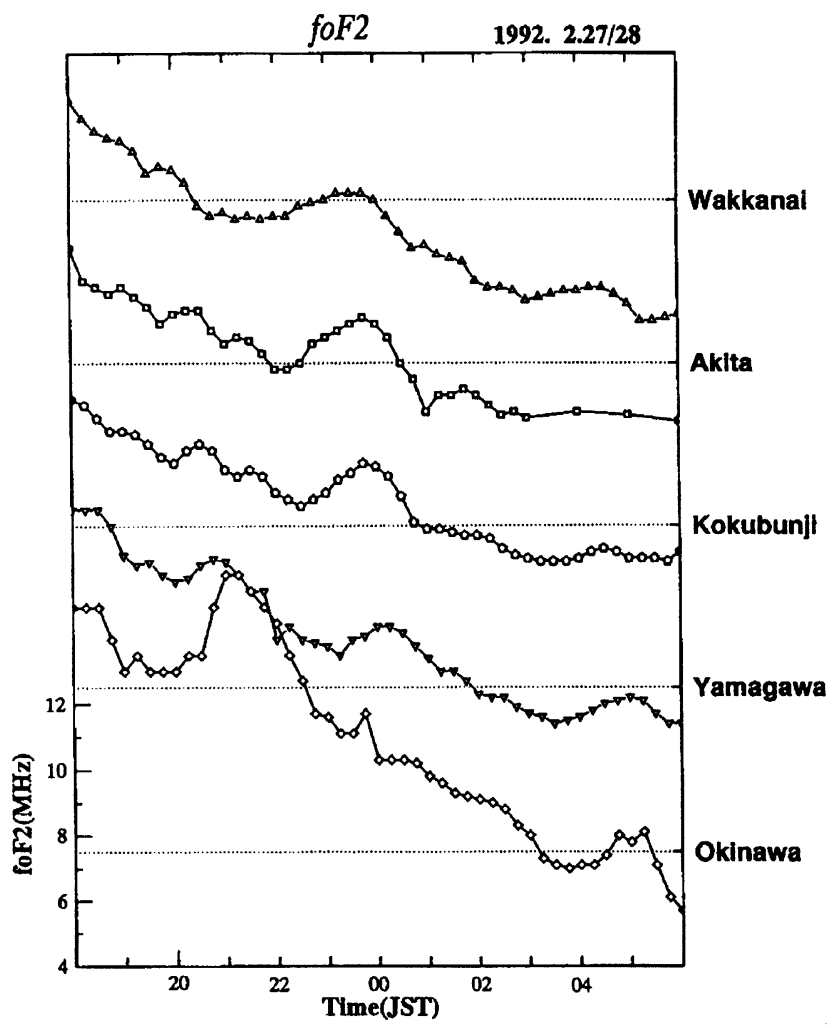

Fig. 14. Variations of $f_{0} F_{2}$ observed at Wakkanai, Akita, Kokubunji, Yamagawa, and Okinawa on February 27/28, 1992.

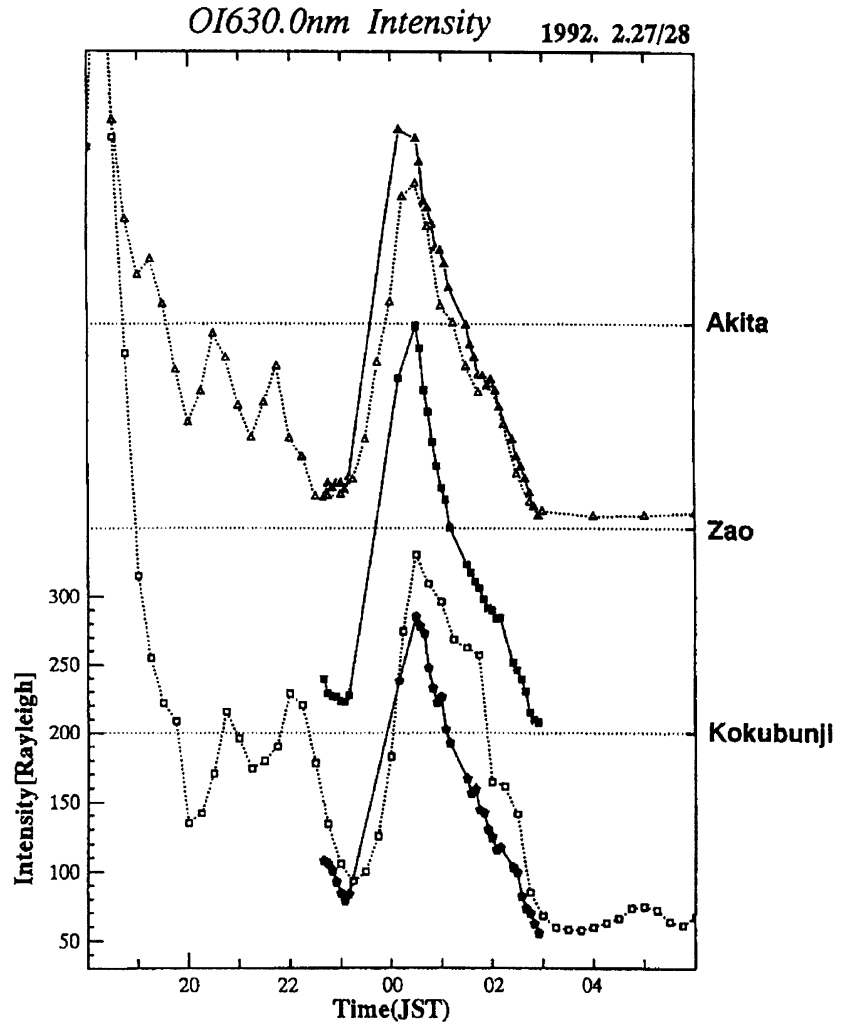

Fig. 15. Variations of OI 630-nm intensity at the zenith of Akita, Zao, and Kokubunji observatories on February 27/28, 1992. Solid lines are intensities obtained with the MAIS, while dotted lines are values calculated from the ionospheric data using the Barbier's formula.

is found that there is a good agreement between the observed and calculated OI 630-nm intensities. In this calculation, we used $41.5 \mathrm{~km}, 10.35$, and 69.3 for the values of $H, A$, and $B$, respectively. The values of $A$ and $B$ on this night are much larger than ones on the night of June $2 / 3$.

Suzuki and Ogawa (1975) derived that the values of $A$ and $B$ are about 2.07 and 30.7 , respectively, when the dissociative recombination (DR) dominates in the excitation processes of the OI $630-\mathrm{nm}$ emission. Because their observational instruments and data analysis methods are different from ours, their observational results cannot be simply compared with our results. Nevertheless, the values of $A$ and $B$ on the night of February $27 / 28$ are too large. It means that the OI 630-nm intensity on this night is unexpectedly strong, and the source of excitation cannot be explained only by the DR excitation process. Shiokawa et al. (1994) reported that low-latitude auroras were observed in Hokkaido at 2000 JST (1100 UT) on the same night. It suggests that the excitation by precipitating electrons effected the OI 630-nm emission intensity over Japan on the night of February 27/28, 1992.

Solid lines and dashed lines in Fig. 13 are the lines connecting peaks and valleys in the values of $h^{\prime} F$ measured at Wakkanai, Akita, and Kokubunji, respectively. Since Wakkanai and Kokubunji are located at almost the same meridian, as seen in Fig. 6, we can obtain the meridional component of propagation velocities of $h^{\prime} F$ disturbances from the gradients of these lines. Time differences between two consecutive dashed lines are regarded as periods of the vari- 

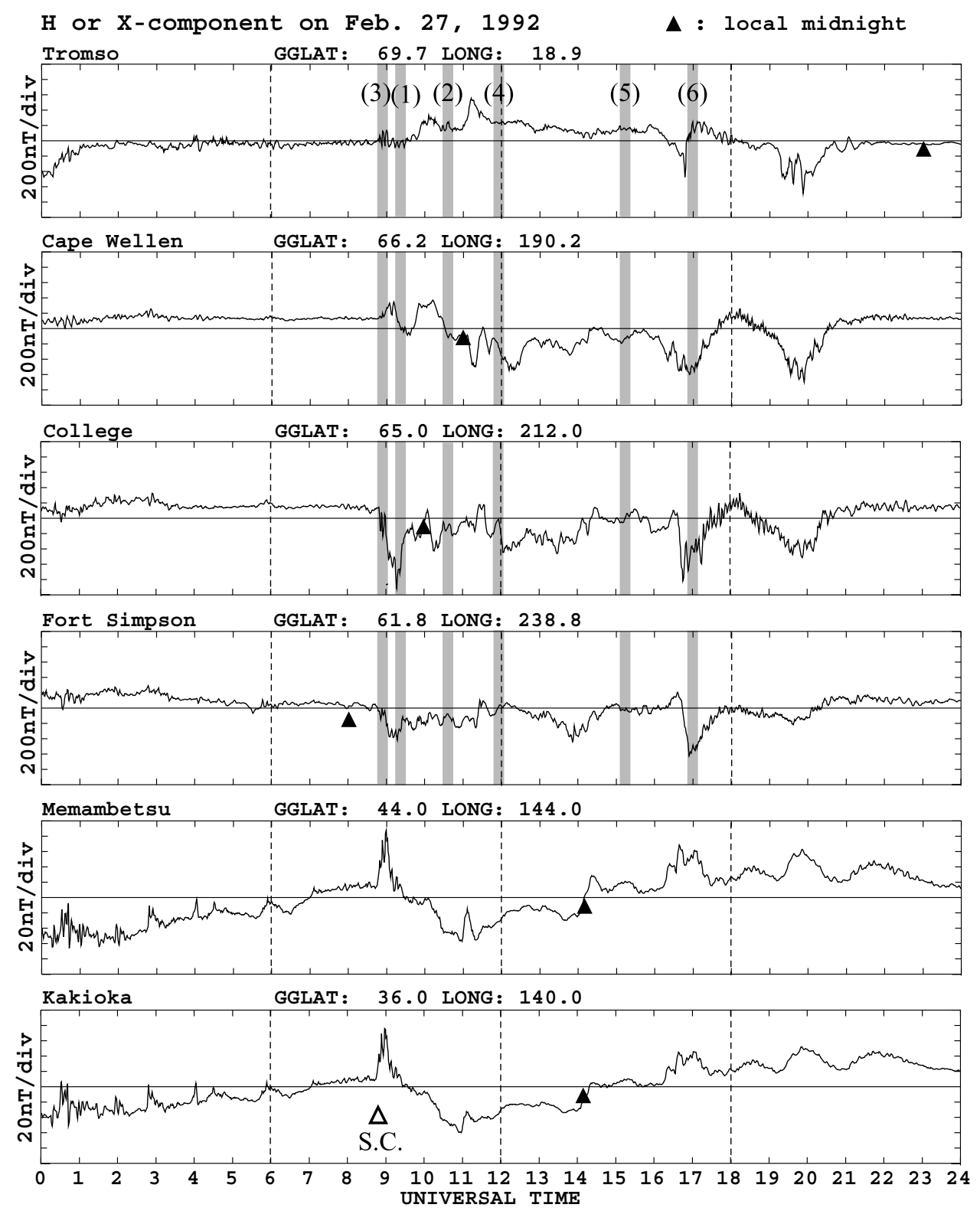

Fig. 16. Magnetograms at high and middle latitudes on February 27, 1992. The horizontal axis is in universal time. Grey thick lines indicate the TID onset times estimated from the ionospheric data shown in Fig. 13. Numbers (1)-(6) correspond to those of wave trains in Fig. 13. (These Geomagnetic data were provided by World Data Center for Geomagnetism, Kyoto University.)

ations. When the period are different from station to station, the value observed at Kokubunji is regarded as the period. Furthermore, horizontal wavelength is calculated from the period and propagation velocity. The values of the meridional component of the propagation velocity, period, and wavelength are thus obtained, as listed in Table 1. This table demonstrates that the disturbances in ionospheric parameters on February 27/28, 1992 can be classified as large-scale TIDs by phase velocities $(305-695 \mathrm{~m} / \mathrm{s})$, wavelengths $(930-5250$ $\mathrm{km})$, and periods (0.7-2.1 hours).

The geomagnetic data on February 27, 1992 are presented in Fig. 16. The time interval of 11-18 UT on February 27, 1992, corresponding to the night of February 27/28 in Japan, geomagnetic activity was considerably high. The preceding sudden commencement of a geomagnetic storm occurred at 1748 JST (0848 UT) on February 27. If we assume that the source region of the large-scale TIDs is located at $67^{\circ} \mathrm{N}$ (magnetic latitude) in the auroral oval, the onset times of the TIDs can be estimated from the meridional distance between the source region and Kokubunji, the time of $h^{\prime} F$ peak at Kokubunji, and propagation velocity. Since, the geomagnetic latitude of Kokubunji is about $26^{\circ} \mathrm{N}$ and the meridional distance between Kokubunji and the source region is about $4500 \mathrm{~km}$. The onset times traced back to the source region in the auroral oval for each peak in the variation of $h^{\prime} F$ are listed in Table 1, and are shown in Fig. 16. The range of the TID onset times agrees with the period of large substorms during the geomagnetic storm (as seen in Fig. 16). It is therefore strongly suggested that the large-scale TIDs observed over Japan on this night were excited by high-latitude substorm processes and that the passage of the TIDs remarkably enhanced the OI 630-nm intensity. 
Table 1. List of phase velocities, periods, and wavelengths of the large-scale TID event on the night of February 27/28, 1992. Numbers (1)-(6) correspond to those of wave trains shown in Fig. 13.

\begin{tabular}{cccccc}
\hline Number & $\begin{array}{c}\text { Period } \\
\text { (hour) }\end{array}$ & $\begin{array}{c}\text { Phase velocity } \\
(\text { S-N component })\end{array}$ & $\begin{array}{c}\text { Wavelength } \\
(\mathrm{m} / \mathrm{s})\end{array}$ & $\begin{array}{c}\text { Time of the peak } \\
\text { at Kokubunji } \\
(\mathrm{km})\end{array}$ & $\begin{array}{c}\text { Time traced back } \\
\text { to the aurora oval } \\
(\text { UT })\end{array}$ \\
\hline$(1)$ & 1.3 & 695 & 3,250 & 2008 & 0920 \\
$(2)$ & 1.0 & 695 & 2,500 & 2123 & 1038 \\
$(3)$ & 3.0 & 305 & 3,300 & 2315 & 0855 \\
$(4)$ & 0.7 & 370 & 930 & 0110 & 1157 \\
$(5)$ & 1.0 & 695 & 2,500 & 0200 & 1515 \\
$(6)$ & 2.1 & 695 & 5,250 & 0345 & 1700 \\
\hline
\end{tabular}

\section{Summary and Conclusions}

Atmospheric gravity waves and TIDs play an important role in the dynamics and energetics in the thermosphere and ionosphere. To understand these wave phenomena, we carried out imaging observations with the MAIS at the Zao observatory, Japan. From these observations, wave phenomena corresponding to medium- and large-scale TIDs were detected in the OI 630-nm nightglow images. These observations are summarized as the followings:

Wave-like structures appeared in the images of the OI 630nm airglow on the night of June 2/3, 1992 during a geomagnetically quiet period $\left(K p=1_{-}-1_{+}\right)$. These waves were identified to be caused by a propagation of the medium-scale TID from both the airglow and ionospheric observations. Phase velocity and horizontal wavelength determined from the OI $630-\mathrm{nm}$ image data on this night are $45-100 \mathrm{~m} / \mathrm{s}$ and $\sim 280 \mathrm{~km}$, respectively, and the propagation direction is south-westward. This is the first observation in which a propagation of the medium-scale TID in the mid-latitude region was detected by an airglow imaging method.

A large enhancement of the OI 630-nm intensity observed on the night of February 27/28, 1992 during a geomagnetic storm was identified to be caused by propagation of largescale TIDs. Meridional components of phase velocities and wavelengths determined from ionospheric data on this night are $305-695 \mathrm{~m} / \mathrm{s}$ (southward), 930-5250 km, respectively. Periods are $0.7-2.1$ hours. The source of these large-scale TIDs is suggested to be substorm processes at high latitudes.

These two events have demonstrate that airglow imagers like the MAIS give useful information about the dynamics and energetics in the thermosphere and ionosphere.

Acknowledgments. The authors would like to acknowledge Toshihiro Abe for his technical support. They also thank Yoshiyuki Kiyama for his useful suggestions and technical support on the calibration of the MAIS. The ionosonde data were provided by World Data Center (WDC-C2) for Ionosphere, Communications Research Laboratory, Tokyo, Japan. Geomagnetic data were provided by World Data Center (WDC-C2) for Geomagnetism, Data Analysis Center for Geomagnetism and Space Magnetism, Graduate School of Science, Kyoto University, Kyoto, Japan.

\section{References}

Arthur, N. C., Allen's astrophysical quantities, fourth edition, pp. 265-270,
Springer-Verlag New York Inc., NY, 2000.

Barbier, D., Recherches sur la raie 6300 de la luminescence atmosphérique nocturne, Ann. Géophys., 15, 179-217, 1959.

Barbier, D. and J. Glaume, La couche ionosphérique nocturne F dans la zone intertropicale et ses relations avec l'émission de la raie $6300 \AA ̊ d u$ ciel nocturne, Planet. Space Sci., 9, 133-149, 1962.

Hajkowicz, L. A., A global study of large scale traveling ionospheric disturbances (TIDs) following a step-like onset of auroral substorms in both hemispheres, Planet. Space Sci., 38, 913-923, 1990.

Hajkowicz, L. A., Auroral electrojet effect on the global occurrence pattern of large scale traveling ionospheric disturbances, Planet. Space Sci., 39, 1189-1196, 1991.

Hines, C. O., Internal atmospheric gravity waves at ionospheric heights, Can. J. Phys., 38, 1441-1481, 1960.

Hunsucker, R. D., Atmospheric gravity waves generated in the high-latitude ionosphere: a review, Rev. Geophys. Space Phys., 20, 293-315, 1982.

Kainuma, S., K. Inamori, H. Ishibashi, T. Isobe, and T. Ogawa, Ionospheric disturbances during November $30 \sim$ December 1, 1988; 5. ionospheric oscillations revealed by HF Doppler measurement technique, J. Commun. Res. Lab., 39, 277-286, 1992.

Kasten, F., A new table and approximate formula for relative optical air mass, Arch. Mateorol. Geophys. Bioklimatol., Ser. B14, 206-223, 1966.

Mendillo, M., J. Baumgardner, D. Nottingham, J. Aarons, B. Reinisch, J. Scali, and M. Kelley, Investigations of thermospheric-ionospheric dynamics with $6300-\AA$ images from the Arecibo Observatory, J. Geophys. Res., 102, 7331-7343, 1997.

Porter, H. S., S. M. Silverman, and T. F. Tuan, On the behavior of airglow under the influence of gravity waves, J. Geophys. Res., 79, 3827-3833, 1974.

Shibata, T., Two classes of medium-scale traveling ionospheric disturbances observed by an HF Doppler Array, J. Geomag. Geoelectr., 38, 779-796, 1986.

Shiokawa, K., K. Yumoto, Y. Tanaka, T. Oguti, and Y. Kiyama, Law-latitude auroras observed at Moshiri and Rikubetsu $(\mathrm{L}=1.6)$ during magnetic storms on February 26, 27, 29, and May 10, 1992, J. Geomag. Geoelectr., 46, 231-252, 1994.

Sobral, J. H. A., H. C. Carlson, D. T. Farley, and W. E. Swartz, Nighttime dynamics of the $\mathrm{F}$ region near Arecibo as mapped by airglow features, $J$. Geophys. Res., 83, 2561-2566, 1978.

Suzuki, K. and T. Ogawa, Twilight OI 6300 A airglow measurement using a high dispersion monochrometor, J. Geomag. Geoelectr., 27, 175-186, 1975.

Taylor, M. J., J.-M. Jahn, S. Fukao, and A. Saito, Possible evidence of gravity wave coupling into the mid-latitude $\mathrm{F}$ region ionosphere during the SEEK campaign, Geophys. Res. Lett., 25, 1801-1804, 1998.

Tohmatsu, T. and T. Ogawa, Compedium of Aeronomy, Terra Scientific Publishing Co., Tokyo, 363 pp., 1990.

Waldock, J. A. and T. B. Jones, Source regions of medium scale traveling ionospheric disturbances observed at middle-latitudes, J. Atmos. Terr. Phys., 49, 105-114, 1987.

M. Kubota (e-mail: mkubota@crl.go.jp), H. Fukunishi, and S. Okano 